\title{
Sedentary Behaviors Of A School Population In Brazil And Related Factors Descriptive Research With Questionnaire
}

José Antonio Ponce-Blandón

Centro de Enfermería Cruz Roja, adscrito a la Universidad de Sevilla

María Eduarda Deitos-Vasquez

Universidade Federal do Pampa

Rocío Romero-Castillo ( $\square$ rocioromerocastillo@gmail.com )

Universidad de Huelva - Campus El Carmen https://orcid.org/0000-0001-9199-030X

Diogo da Rosa-Viana

Universidade Federal do Rio Grande do Sul Instituto de Ciencias Basicas da Saude

José Miguel Robles-Romero

Centro de Enfermería Cruz Roja, adscrito a la Universidad de Sevilla

Jussara Mendes-Lipinski

Universidade Federal do Pampa

Research

Keywords: Feeding Behavior, Healthy Lifestyle, Sedentary Behavior, Exercise, Child, Child Behavior, Schools.

Posted Date: June 2nd, 2020

DOl: https://doi.org/10.21203/rs.3.rs-32130/v1

License: (9) This work is licensed under a Creative Commons Attribution 4.0 International License. Read Full License 


\section{Abstract}

Background Overweight and obesity arise from a complex range of genetic, environmental, behavioural, educational, and socioeconomic factors. Poor eating habits and poor physical activity increase the probability that children will become obese adolescents. The sedentary lifestyle, encouraged by the rapid evolution of technology, has been accentuated in recent years. Strategies to accelerate obesity prevention include modifying the child's environment for healthy eating and physical activity. For this, it is essential to analyze the characteristics and life habits of children population. This study tries to know the sedentary practices and some life habits related to health among children from the school population of Uruguaiana (RS, Brazil), identifying the sociodemographic factors that can influence it.

Methods The sample was composed of 470 school boys and girls, aged between 9 and 10 years. In order to obtain the sample, a random plampling was performed by clusters of the 24 urban public schools of the city. Sociodemographic variables and sedentary habits were measured.

Results As regards the variables linked to habits, $24 \%$ of the boys and girls answered they had not had breakfast the day they completed the questionnaire. $51.8 \%$ stated they did not have breakfast any given day of the week. Regarding to sedentary habits, $25.3 \%$ of children watched TV or played video games 5 or more hours a day and $9 \%$ rarely played sports with their parents or caregivers. Statistical significance was recorded between "number of hours watching TV and playing video games" and "playing sports with parents or caregivers" $(p<0.05)$.

Conclusions Association between the times spent watching TV or playing video games and the practice of physical exercise in the family, proves once again the importance of the family in education for the health of children. The school provides direct Access to schoolchildren and their parents to launch numerous health education programs.

\section{Background}

According to the World Health Organization, childhood obesity is a Public Health problem, whose prevalence has increased in recent years [1]. If current trends continue, in 2022 there will be more children with obesity than with underweight [2]. Overweight and obesity in children cause significant impacts on the physical and mental health of children, with a tendency towards obesity in adulthood and suffering from non-communicable diseases such as diabetes and cardiovascular diseases [3, 4] In Latin America, it is estimated that between 42.4 and 51.8 million children and adolescents are overweight or obese, representing 20 to $25 \%$ of the total population of children and adolescents [5]. Specifically in Brazil, one third of children population between 5 to 9 years old is overweight [6].

Overweight and obesity arise from a complex range of genetic, environmental, behavioural, educational, and socioeconomic factors. Poor eating habits and poor physical activity increase the probability that children will become obese adolescents [7]. The sedentary lifestyle, encouraged by the rapid evolution of technology, has been accentuated in recent years [8]. Life habits have been modified especially in 
children, who have adopted behaviours that predispose to minimal energy expenditure, close to a quiescent condition. Children spend many hours in front of television, playing video games or managing social networks from their mobile phones [9]. According to a survey carried out in Brazil, entitled National Student Health Survey, the prevalence of adolescents exposed to at least two hours a day of television is $78 \%[10]$.

There is scientific evidence highlighting the association between the number of hours that television is viewed and the omission of important meals of the day such as breakfast, and also with the consumption of hypercaloric foods, high in sugars, carbohydrates and saturated fats $[4,11]$. The omission of breakfast has also been linked to a higher body mass index and an increase in leisure time in sedentary activities [12]. One of the strategies to control obesity in Brazil is through the National Food and Nutrition Policy (PNAN), updated by Ordinance No. 2,715 / 2011, which aims to improve the diet, nutrition and health conditions of Brazilian population, through actions in order to promote adequate and healthy eating practices, food and nutritional surveillance, especially the prevention of health problems related to an inadequate diet [13].

Strategies to accelerate obesity prevention include modifying the child's environment for healthy eating and physical activity [14]. For this, it is essential to analyze the characteristics and life habits of children population. This study tries to know the sedentary practices and some life habits related to health among children from the school population of Uruguaiana (RS, Brazil), identifying the sociodemographic factors that can influence it. The main hypothesis of the study is that the exercise with the family reduces the hours of exposure to television and video games in children.

\section{Methods}

A cross-sectional descriptive study was conducted to characterize the life habits of the school boys and girls from 4th grade (9-10 years old) in the city of Uruguaiana (Rio Grande do Sul, Brazil). In order to obtain the sample, a random sampling was performed by clusters of the 24 urban public schools of the city. In the random selection of clusters, 12 schools were included with a total of 558 4th grade boys and girls enrolled, so as to guarantee a minimum sampling size of 264 boys and girls an 11 clusters, considering an acceptable marginal error of $5 \%$ and a confidence level of $95 \%$.

A transcultural adaptation was conducted of the abridged questionnaire of Eating Habits of the School Population of the "PERSEO" program, by the Spanish Society of Community Nutrition, validated in the Spanish population [15]. A draft was sent to three social scientists and experts in this area that are external to the research team. They performed a review of the translated questionnaire, considering aspects such as item comprehension (both questions and possible answers) in relation to the objectives of the research. The items related to sedentary habits were selected. Questions include single, multiple, and open answers that are grouped in different variables:

- Sociodemographic variables: age, gender, family situation, the parents' working situation and their type occupation, according to the Brazilian classification of occupations [16]. 
- Variables of sedentary habits: number of hours watching television, videos or playing videogames, television watching frequency during lunch or dinner, sports activities with parents.

The questionnaire was applied to the selected sample in 12 schools. The headships of all the selected school centres were contacted. The data collection day and time were scheduled and the boys' and girls' parents were asked to sign the informed consent. Two educated researchers, accompanied by the teacher of the group, explained the instructions to complete the questionnaire to the children and clarified any doubt. To record and analyse the data, a questionnaire was written in the specific tool from the Epilnfo application, version 7.2.1.0. This tool was also employed to perform the statistical analysis.

The frequency distributions of the main qualitative variables were calculated in the descriptive analysis, as well as the measurements of central tendency and dispersion of the quantitative variables. For the hypothesis contrast, the Chi-square test or Fisher's exact test and Student's $t$ test were used, as it suits the case. The accepted statistical significance level for the hypothesis contrast was set at $p<0.05$.

The research obtained documentary authorization from the Regional Coordinating Office for Education of Rio Grande do Sul and from the Research Ethics Committee of the University of Unipampa. During the whole process of data collection, children's anonymity was guaranteed and the ethical principles for medical research in human beings described in the latest revision of the Declaration of Helsinki were applied [17].

\section{Results}

A total of 4704 th grade boys and girls (9-10 years old) participated in the study, according to the distribution of the participating centres of the city shown in Table 1. Of the total of participants, $46 \%$ were boys $(n=196 ; 95 \% \mathrm{Cl}[41.3-50.7])$ and $54 \%$ were girls $(n=230 ; 95 \% \mathrm{Cl}[49.2-58.6])$ and the mean age of the participating children was 9.8 years old $(S D=0.74) .38 \%(n=173 ; 95 \% \mathrm{Cl}[33.7-42.5])$ were children from the morning group, $56.7 \%(n=258 ; 95 \% \mathrm{Cl}$ [52.1-61.2]) were from the afternoon group, and 5.3\% ( $n$ $=24 ; 95 \% \mathrm{Cl}[3.6-7.7]$ ) were from the integrative group (morning and afternoon shifts). $99.6 \%$ of the children were Brazilian ( $n=468 ; 95 \% \mathrm{Cl}[98.4-99.8]) .64 .2 \%$ of the children (95\% Cl [64.2-92.9]) stated they lived with their parents all the time, whereas the rest had different family situations. 
Table 1

Sample distribution by participating centers

\begin{tabular}{|c|c|c|c|c|}
\hline CENTER & $\begin{array}{l}\mathrm{N} \\
\text { (Enrolled } \\
\text { Students) }\end{array}$ & $\begin{array}{l}N \text { (Participating } \\
\text { Students in the } \\
\text { simple) }\end{array}$ & $\begin{array}{l}\text { \% over the } \\
\text { total } \\
\text { sample }\end{array}$ & $\begin{array}{l}\text { IC } \\
95 \%\end{array}$ \\
\hline $\begin{array}{l}\text { Escola Estadual de Ensino Médio } \\
\text { "Dom Hermeto" }\end{array}$ & 140 & 125 & $27,41 \%$ & $\begin{array}{l}{[23} \\
5-31 \\
6]\end{array}$ \\
\hline $\begin{array}{l}\text { Instituto Estadual de Educação "Elisa } \\
\text { Ferrari Valls" }\end{array}$ & 19 & 15 & $3,29 \%$ & $\begin{array}{l}{[2,0-} \\
5,3]\end{array}$ \\
\hline $\begin{array}{l}\text { Escola Estadual de Ensino } \\
\text { Fundamental "Adir Mascia" }\end{array}$ & 73 & 64 & $14,04 \%$ & $\begin{array}{l}{[11} \\
1-17 \\
5]\end{array}$ \\
\hline $\begin{array}{l}\text { Escola Estadual de Ensino } \\
\text { Fundamental "Antonio Mary Ulrich" }\end{array}$ & 32 & 24 & $5,26 \%$ & $\begin{array}{l}{[3,5-} \\
7,7]\end{array}$ \\
\hline $\begin{array}{l}\text { Escola Estadual de Ensino } \\
\text { Fundamental "Iris Valls" }\end{array}$ & 34 & 30 & $6,58 \%$ & $\begin{array}{l}{[4,6-} \\
9,2]\end{array}$ \\
\hline $\begin{array}{l}\text { Escola Estadual de Ensino } \\
\text { Fundamental "Paso de Los Libres" }\end{array}$ & 26 & 23 & $5,04 \%$ & $\begin{array}{l}{[3,3-} \\
7,4]\end{array}$ \\
\hline Instituto Estadual "Romaguera Correa” & 20 & 17 & $3,73 \%$ & $\begin{array}{l}{[2,3-} \\
5,9]\end{array}$ \\
\hline $\begin{array}{l}\text { Escola Estadual de Ensino } \\
\text { Fundamental "Hermeto Jose Pinto } \\
\text { Bermudez" }\end{array}$ & 51 & 37 & $8,11 \%$ & $\begin{array}{l}{[5,9-} \\
10,9]\end{array}$ \\
\hline $\begin{array}{l}\text { Escola Estadual de Ensino Médio } \\
\text { "Senador Salgado Filho" }\end{array}$ & 26 & 23 & $5,04 \%$ & $\begin{array}{l}{[3,3-} \\
7,4]\end{array}$ \\
\hline $\begin{array}{l}\text { Escola Estadual de Ensino Médio } \\
\text { "Professora Lilia Guimaraes" }\end{array}$ & 54 & 42 & $9,21 \%$ & $\begin{array}{l}{[6,8-} \\
12,2]\end{array}$ \\
\hline $\begin{array}{l}\text { Colégio Estadual “Dr Roberval } \\
\text { Beheregaray Azevedo" }\end{array}$ & 46 & 32 & $7,02 \%$ & $\begin{array}{l}{[5,0-} \\
9,7]\end{array}$ \\
\hline $\begin{array}{l}\text { Escola Estadual de Ensino Médio } \\
\text { "Embaixador Joao Baptista Lusardo" }\end{array}$ & 37 & 24 & $5,26 \%$ & $\begin{array}{l}{[3,5-} \\
7,7]\end{array}$ \\
\hline Total & 558 & 456 & $100,00 \%$ & \\
\hline
\end{tabular}

The mean number of brothers/sisters reported by the children was $2.07(\mathrm{SD}=1.48) .83 .5 \%(95 \% \mathrm{Cl}[79.8-$ 86.6]) of the children stated that their father worked, and $63.7 \%(95 \% \mathrm{Cl}$ [59.2-68.0]) that their mother also worked. The main occupation of $51.4 \%(95 \% \mathrm{Cl}$ [46.2-56.7]) of the mothers who worked was in the area of services and in retail sales in stores or supermarkets. $77.1 \%$ of the fathers (95\% $\mathrm{Cl}$ [68.1-82.3]) shared this same occupation. The main sociodemographic and characteristics descriptive results of the sample are summarized in Table 2. 
Table 2

Sociodemographic and characteristics of the sample

\begin{tabular}{|c|c|c|c|c|}
\hline Variables & $\mathbf{n}$ & Media & \multicolumn{2}{|c|}{$\begin{array}{l}\text { Standard } \\
\text { Deviation }\end{array}$} \\
\hline Age (years) & 423 & 9,8 & \multicolumn{2}{|l|}{0,74} \\
\hline $\begin{array}{l}\text { Number of } \\
\text { brothers or } \\
\text { sisters }\end{array}$ & 318 & 2,07 & \multicolumn{2}{|l|}{1,48} \\
\hline $\begin{array}{l}\text { Qualitative } \\
\text { variables }\end{array}$ & Categories & $\mathbf{n}$ & $\%$ & $\begin{array}{l}\text { IC } \\
95 \%\end{array}$ \\
\hline \multirow[t]{2}{*}{ Sex } & Women & 230 & 54,0 & $\begin{array}{l}{[49,2-} \\
58,6]\end{array}$ \\
\hline & Mens & 196 & 46,0 & $\begin{array}{l}{[41,3-} \\
50,7]\end{array}$ \\
\hline \multirow[t]{3}{*}{ Class } & Morning class & 173 & 38,0 & $\begin{array}{l}{[33,7-} \\
42,5]\end{array}$ \\
\hline & Afternoon class & 258 & 56,7 & $\begin{array}{l}{[52,1-} \\
61,2]\end{array}$ \\
\hline & Integral class & 24 & 5,3 & $\begin{array}{l}{[3,6-} \\
7,7]\end{array}$ \\
\hline \multirow[t]{2}{*}{ Nationality } & Brasil & 468 & 99,6 & $\begin{array}{l}{[98,4-} \\
99,8]\end{array}$ \\
\hline & Others (Argentina, Uruguay) & 2 & 0,4 & $\begin{array}{l}{[0,04} \\
-1,2]\end{array}$ \\
\hline \multirow[t]{6}{*}{$\begin{array}{l}\text { Family living } \\
\text { situation }\end{array}$} & Alone with the mother & 77 & 17,6 & $\begin{array}{l}{[14,} \\
3-21, \\
5]\end{array}$ \\
\hline & Alone with the father & 19 & 4,3 & $\begin{array}{l}{[2,8-} \\
6,7]\end{array}$ \\
\hline & With the mother and her new partner & 20 & 4,6 & $\begin{array}{l}{[2,9-} \\
6,9]\end{array}$ \\
\hline & With the father and his new partner & 9 & 2,06 & $\begin{array}{l}{[1,0-} \\
3,8]\end{array}$ \\
\hline & With father and mother & 280 & 64,2 & $\begin{array}{l}{[59,6-} \\
68,5]\end{array}$ \\
\hline & With other adults & 31 & 7,1 & $\begin{array}{l}{[5,0-} \\
9,9]\end{array}$ \\
\hline $\begin{array}{l}\text { Does your } \\
\text { father work? }\end{array}$ & Yes, he works & 375 & 83,5 & $\begin{array}{l}{[79,8-} \\
86,6]\end{array}$ \\
\hline
\end{tabular}


n

Media

Standard

Deviation

\begin{tabular}{|c|c|c|c|c|}
\hline & He doesn't work & 30 & 6,7 & $\begin{array}{l}{[4,7-} \\
9,4]\end{array}$ \\
\hline & I don't know & 22 & 4,9 & $\begin{array}{l}{[3,2-} \\
7,3]\end{array}$ \\
\hline & I haven't contact with my father & 22 & 4,9 & $\begin{array}{l}{[3,2-} \\
7,3]\end{array}$ \\
\hline \multirow{9}{*}{$\begin{array}{l}\text { Type of } \\
\text { father's } \\
\text { occupation }\end{array}$} & 0 Armed forces, Police, fire and military & 1 & 0,2 & $\begin{array}{l}{[-0,21} \\
-0,6]\end{array}$ \\
\hline & $\begin{array}{l}1 \text { Senior members of the government, managers of } \\
\text { public interest organizations and companies and } \\
\text { managers }\end{array}$ & 1 & 0,2 & $\begin{array}{l}{[-0,21} \\
-0,6]\end{array}$ \\
\hline & 2 Science and arts professionals & 8 & 1,8 & $\begin{array}{l}{[1,5-} \\
2,3]\end{array}$ \\
\hline & 3 Level technicians & 11 & 2,4 & $\begin{array}{l}{[1-3,} \\
8]\end{array}$ \\
\hline & 4 Administrative service workers & 25 & 5,6 & $\begin{array}{l}{[3,4-} \\
7,6]\end{array}$ \\
\hline & $\begin{array}{l}5 \text { Service workers, retail salespeople in stores and } \\
\text { markets }\end{array}$ & 199 & 44,2 & $\begin{array}{l}{[39,6-} \\
48,8]\end{array}$ \\
\hline & 6 Agricultural, forestry, hunting and fishing workers & 0 & 0,0 & $\begin{array}{l}{[0,0-} \\
0,0]\end{array}$ \\
\hline & 7 Industrial and service production workers & 6 & 1,3 & $\begin{array}{l}{[0,3-} \\
2,4]\end{array}$ \\
\hline & 8 Maintenance and repair workers & 3 & 0,7 & $\begin{array}{l}{[-0,1-} \\
1,4]\end{array}$ \\
\hline \multirow[t]{4}{*}{$\begin{array}{l}\text { Does your } \\
\text { mother work? }\end{array}$} & Yes, she works & 288 & 63,7 & $\begin{array}{l}{[57,9-} \\
66,9]\end{array}$ \\
\hline & She doesn't work & 144 & 31,8 & $\begin{array}{l}{[27,} \\
7-36, \\
3]\end{array}$ \\
\hline & I don't know & 14 & 3,1 & $\begin{array}{l}{[1,5-} \\
4,7]\end{array}$ \\
\hline & I haven't contact with my mother & 6 & 1,3 & $\begin{array}{l}{[0,3-} \\
2,4]\end{array}$ \\
\hline $\begin{array}{l}\text { Type of } \\
\text { mother's } \\
\text { occunation }\end{array}$ & 0 Armed forces, Police, fire and military & 10 & 2,2 & $\begin{array}{l}{[0,8-} \\
3,6]\end{array}$ \\
\hline
\end{tabular}

Type of

father's

occupation

Does your

mother work?

occupation 


\begin{tabular}{|c|c|c|c|c|}
\hline \multirow[t]{2}{*}{ Variables } & \multirow{2}{*}{$\begin{array}{l}\text { n } \\
\begin{array}{l}1 \text { Senior members of the government, managers of } \\
\text { public interest organizations and companies and } \\
\text { managers }\end{array}\end{array}$} & \multirow{2}{*}{$\begin{array}{l}\text { Media } \\
3\end{array}$} & \multicolumn{2}{|c|}{$\begin{array}{l}\text { Standard } \\
\text { Deviation }\end{array}$} \\
\hline & & & 0,7 & $\begin{array}{l}{[0,1-} \\
1,4]\end{array}$ \\
\hline & 2 Science and arts professionals & 5 & 1,1 & $\begin{array}{l}{[0,1-} \\
2,1]\end{array}$ \\
\hline & 3 Level technicians & 12 & 2,7 & $\begin{array}{l}{[1,2-} \\
4,1]\end{array}$ \\
\hline & 4 Administrative service workers & 25 & 5,6 & $\begin{array}{l}{[3,4-} \\
7,7]\end{array}$ \\
\hline & $\begin{array}{l}5 \text { Service workers, retail salespeople in stores and } \\
\text { markets }\end{array}$ & 175 & 38,9 & $\begin{array}{l}{[34,4-} \\
43,4]\end{array}$ \\
\hline & 6 Agricultural, forestry, hunting and fishing workers & 18 & 4,0 & $\begin{array}{l}{[2,2-} \\
5,8]\end{array}$ \\
\hline & 7 Industrial and service production workers & 41 & 9,1 & $\begin{array}{l}{[6,4-} \\
11,8]\end{array}$ \\
\hline & 8 Maintenance and repair workers & 49 & 10,9 & $\begin{array}{l}{[8,0-} \\
13,8]\end{array}$ \\
\hline
\end{tabular}

As regards the variables linked to habits, referred to in Table 3, 24\% (95\% Cl [18.8-28.2]) of the boys and girls answered they had not had breakfast the day they completed the questionnaire. $34.9 \%(95 \% \mathrm{Cl}$ [30.5-39.3]) who have breakfast had taken milk, eaten legumes, dried fruits or eggs, $24.4 \%(95 \% \mathrm{Cl}$ [20.5-28.4]) fruits or natural fruit juices, and 10.4\% (95\% $\mathrm{Cl}$ [7.6-13.3]) bread, rice or potatoes, $6.9 \%$ (95\% $\mathrm{Cl}[4.5-9.3])$ cold meats and processed foods and $3.1 \%(95 \% \mathrm{Cl}$ [1.5-4.7]) candies, pastries or salted snacks. With respect to the frequency of weekly breakfast $51.8 \%$ (95\% Cl [47.1-56.4]) stated they did not have breakfast any given day of the week. $49.3 \%$ (95\% $\mathrm{Cl}$ [44.7-54.0]) had breakfast every day with their mother or father and $14.2 \%(95 \% \mathrm{Cl}$ [10.9-17.5]) never had breakfast with their mother/father. Regarding to sedentary habits, $25.3 \%(95 \% \mathrm{Cl}$ [21.4-29.3]) of children watched TV or played video games 5 or more hours a day, 55.3\% (95\% Cl [50.8-59.8]) watched TV at lunch and dinner and 9,1\% (95\% Cl [6.8-11.8]) rarely played sports with their parents or caregivers. 
Table 3

Habits of the sample

\begin{tabular}{|c|c|c|c|c|}
\hline Variables & Categories & $\mathbf{n}$ & $\%$ & $\begin{array}{l}\text { IC } \\
95 \%\end{array}$ \\
\hline \multirow[t]{2}{*}{$\begin{array}{l}\text { Have you had breakfast today in the morning } \\
\text { (before coming to school)? }\end{array}$} & Yes & 342 & 76,0 & $\begin{array}{l}{[72,5-} \\
79,5]\end{array}$ \\
\hline & No & 108 & 24,0 & $\begin{array}{l}{[18,} \\
8-28, \\
2]\end{array}$ \\
\hline \multirow[t]{5}{*}{ Breakfast classification } & $\begin{array}{l}\text { Sweets, pastries and } \\
\text { salty snacks }\end{array}$ & 14 & 3,1 & $\begin{array}{l}{[1,50} \\
-4,7]\end{array}$ \\
\hline & $\begin{array}{l}\text { Red, processed and } \\
\text { could meats }\end{array}$ & 31 & 6,9 & $\begin{array}{l}{[4,5-} \\
9,3]\end{array}$ \\
\hline & $\begin{array}{l}\text { Milk, lean meats, } \\
\text { legumes, nuts, eggs }\end{array}$ & 157 & 34,9 & $\begin{array}{l}{[30,} \\
5-39, \\
3]\end{array}$ \\
\hline & Vegetables and fruits & 110 & 24,4 & $\begin{array}{l}{[20,} \\
5-28, \\
4]\end{array}$ \\
\hline & $\begin{array}{l}\text { Bread, pasta, rice, } \\
\text { potatoes }\end{array}$ & 47 & 10,4 & $\begin{array}{l}{[7,6-} \\
13,3]\end{array}$ \\
\hline \multirow[t]{5}{*}{$\begin{array}{l}\text { How often do you have breakfast with your } \\
\text { mother and/or your father? }\end{array}$} & Everyday & 222 & 49,3 & $\begin{array}{l}{[44,7-} \\
54,0]\end{array}$ \\
\hline & $4-6$ days per week & 29 & 6,4 & $\begin{array}{l}{[4,2-} \\
8,7]\end{array}$ \\
\hline & $1-3$ days per week & 66 & 14,7 & $\begin{array}{l}{[11,} \\
4-17, \\
9]\end{array}$ \\
\hline & Less than once a week & 50 & 11,1 & $\begin{array}{l}{[8,2-} \\
14,0]\end{array}$ \\
\hline & Never & 64 & 14,2 & $\begin{array}{l}{[10,} \\
9-17, \\
5]\end{array}$ \\
\hline \multirow[t]{3}{*}{ How many days a week do you have breakfast? } & Everyday & 81 & 18,0 & $\begin{array}{l}{[14,} \\
4-21, \\
6]\end{array}$ \\
\hline & $5-6$ days per week & 81 & 18,0 & $\begin{array}{l}{[14,} \\
4-21, \\
6]\end{array}$ \\
\hline & $1-4$ days per week & 31 & 6,9 & $\begin{array}{l}{[4,5-} \\
9,2]\end{array}$ \\
\hline
\end{tabular}




\begin{tabular}{|c|c|c|c|c|}
\hline Variables & Categories & n & $\%$ & $\begin{array}{l}\text { IC } \\
95 \%\end{array}$ \\
\hline & None & 233 & 51,8 & $\begin{array}{l}{[47,1-} \\
56,4]\end{array}$ \\
\hline \multirow[t]{6}{*}{$\begin{array}{l}\text { How many hours a day you watch television, } \\
\text { videos or do you play video games? }\end{array}$} & None & 47 & 10,0 & $\begin{array}{l}{[7.3-} \\
12.7]\end{array}$ \\
\hline & 1 hour/day & 145 & 30,9 & $\begin{array}{l}{[26.6-} \\
35.1]\end{array}$ \\
\hline & 2 hours/day & 56 & 11,9 & $\begin{array}{l}{[8.9-} \\
14.8]\end{array}$ \\
\hline & 3 hours/day & 44 & 9,4 & $\begin{array}{l}{[6.7-} \\
12]\end{array}$ \\
\hline & 4 hours/day & 39 & 8,3 & $\begin{array}{l}{[5.8-} \\
10.8]\end{array}$ \\
\hline & 5 or more hours/day & 119 & 25,3 & $\begin{array}{l}{[21.4-} \\
29.3]\end{array}$ \\
\hline \multirow[t]{5}{*}{$\begin{array}{l}\text { How often do you watch television at lunch or } \\
\text { dinner at home? }\end{array}$} & Everyday & 260 & 55,3 & $\begin{array}{l}{[50.8-} \\
59.8]\end{array}$ \\
\hline & 4-6 days/week & 41 & 8,7 & $\begin{array}{l}{[6.1-} \\
11.3]\end{array}$ \\
\hline & 1-3 days/week & 64 & 13,6 & $\begin{array}{l}{[10.5-} \\
16.7]\end{array}$ \\
\hline & Less than 1 day/week & 80 & 17,0 & $\begin{array}{l}{[13.6-} \\
20.4]\end{array}$ \\
\hline & Never & 0 & 0,0 & {$[0,0]$} \\
\hline \multirow[t]{5}{*}{$\begin{array}{l}\text { Do you usually do sports with your mother, father } \\
\text { or with a caregiver or relative? }\end{array}$} & Yes, most days & 174 & 37,0 & $\begin{array}{l}{[32.6-} \\
41.4]\end{array}$ \\
\hline & Sometimes & 139 & 29,6 & $\begin{array}{l}{[25.4-} \\
33.7]\end{array}$ \\
\hline & Only on weekends & 34 & 7,2 & $\begin{array}{l}{[4.9-} \\
9.6]\end{array}$ \\
\hline & Rarely & 43 & 9,1 & $\begin{array}{l}{[6.8-} \\
11.8]\end{array}$ \\
\hline & Never & 0 & 0,0 & {$[0,0]$} \\
\hline
\end{tabular}

The results related to the hypothesis contrast, obtained with the Chi-square test, are presented in Table 4. The variable "number of hours watching TV and playing video games" became a dichotomous variable: less than 2 hours and 2 or more hours watching TV or playing videogames. In comparison with other variables (sex, family situation, parents' works and breakfast habits), only statistical significance was 
recorded with two variables. There was a significant difference between number of hours watching TV and playing video games and sex $(p<0.05)$ and playing sports with parents or caregivers $(p<0.05)$. 
Table 4

Contingency table using the chi-squared test

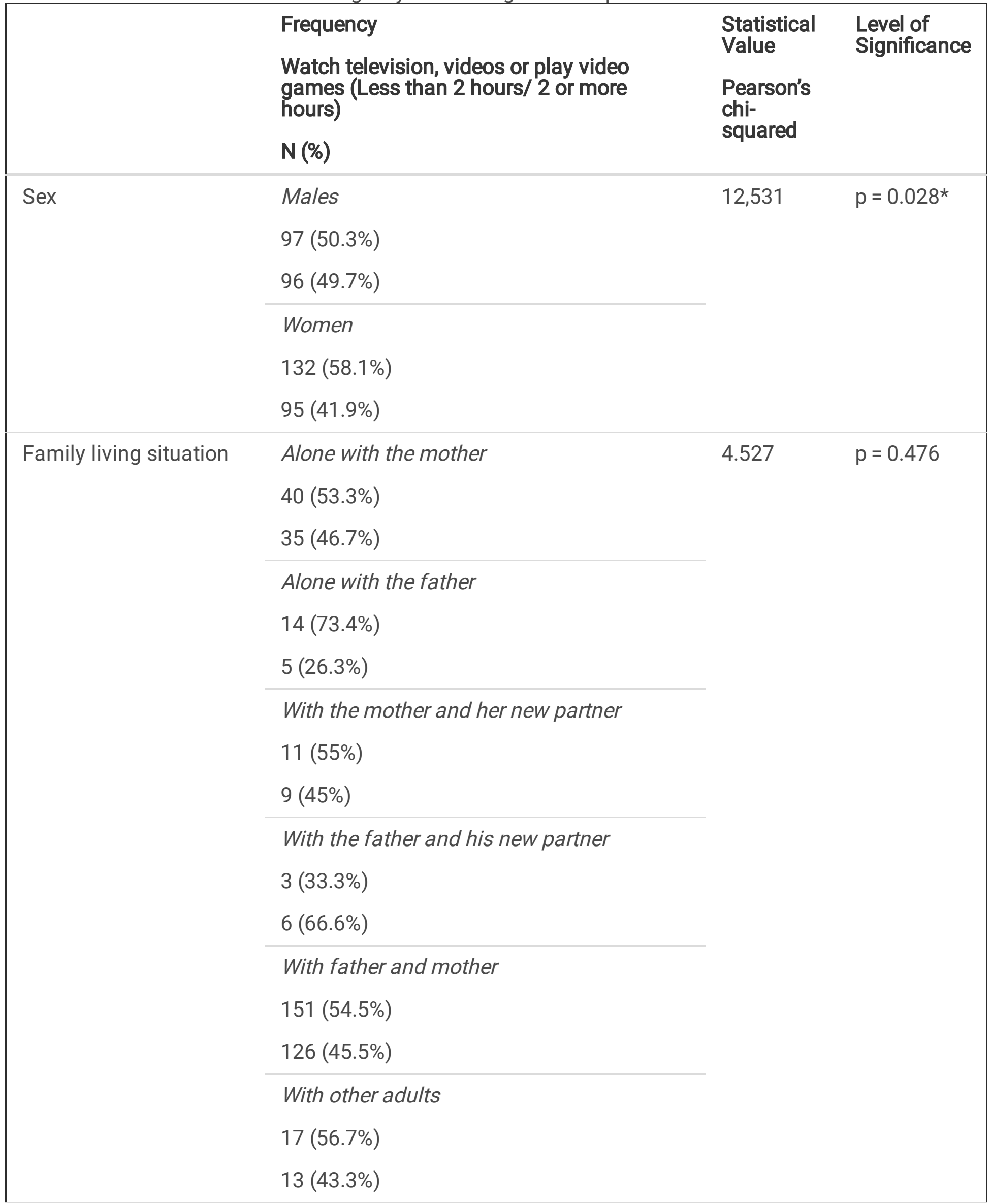




\begin{tabular}{|c|c|c|c|}
\hline & & $\begin{array}{l}\text { Statistical } \\
\text { Value }\end{array}$ & $\begin{array}{l}\text { Level of } \\
\text { Significance }\end{array}$ \\
\hline & $\begin{array}{l}\text { Watch television, videos or play video } \\
\text { games (Less than } 2 \text { hours/ } 2 \text { or more } \\
\text { hours) }\end{array}$ & $\begin{array}{l}\text { Pearson's } \\
\text { chi- }\end{array}$ & \\
\hline & $\mathrm{N}(\%)$ & & \\
\hline Does your father work? & Yes, he works & 1.964 & $p=0.580$ \\
\hline & $198(53.4 \%)$ & & \\
\hline & $173(46.6 \%)$ & & \\
\hline & He doesn't work & & \\
\hline & $17(56.7 \%)$ & & \\
\hline & $13(43.3 \%)$ & & \\
\hline & I don't know & & \\
\hline & $13(61.9 \%)$ & & \\
\hline & $8(38.1 \%)$ & & \\
\hline & I haven't contact with my father & & \\
\hline & $14(66.6 \%)$ & & \\
\hline & 7 (33.3\%) & & \\
\hline Does your mother work? & Yes, she works & 3.977 & $p=0.264$ \\
\hline & $163(57.2 \%)$ & & \\
\hline & $122(42.8 \%)$ & & \\
\hline & She doesn't work & & \\
\hline & $70(49 \%)$ & & \\
\hline & $73(51 \%)$ & & \\
\hline & I don't know & & \\
\hline & $7(58.3 \%)$ & & \\
\hline & $5(41.7 \%)$ & & \\
\hline & I haven't contact with my mother & & \\
\hline & $4(80 \%)$ & & \\
\hline & $1(20 \%)$ & & \\
\hline
\end{tabular}




\begin{tabular}{|c|c|c|c|}
\hline & Frequency & \multirow{3}{*}{$\begin{array}{l}\text { Statistical } \\
\text { Value } \\
\text { Pearson's } \\
\text { chi- } \\
\text { squared }\end{array}$} & \multirow{3}{*}{$\begin{array}{l}\text { Level of } \\
\text { Significance }\end{array}$} \\
\hline & $\begin{array}{l}\text { Watch television, videos or play video } \\
\text { games (Less than } 2 \text { hours/ } 2 \text { or more } \\
\text { hours) }\end{array}$ & & \\
\hline & $\mathbf{N}(\%)$ & & \\
\hline \multirow{15}{*}{$\begin{array}{l}\text { Breakfast frequency } \\
\text { with parents }\end{array}$} & Everyday & 7.131 & $p=0.129$ \\
\hline & $124(56.4 \%)$ & & \\
\hline & $96(43.6 \%)$ & & \\
\hline & 4-6 days per week & & \\
\hline & $22(66.7 \%)$ & & \\
\hline & $11(33.3 \%)$ & & \\
\hline & 1-3 days per week & & \\
\hline & $38(56.7 \%)$ & & \\
\hline & $29(43.3 \%)$ & & \\
\hline & Less than once a week & & \\
\hline & $32(61.5 \%)$ & & \\
\hline & $20(38.5 \%)$ & & \\
\hline & Never & & \\
\hline & $27(42.2 \%)$ & & \\
\hline & 37 (57.8\%) & & \\
\hline \multirow{9}{*}{$\begin{array}{l}\text { How many days a week } \\
\text { do you have breakfast }\end{array}$} & Everyday & 0.270 & $p=0.966$ \\
\hline & $128(53.8 \%)$ & & \\
\hline & $110(46.2 \%)$ & & \\
\hline & 5-6 days/week & & \\
\hline & $18(54.5 \%)$ & & \\
\hline & $15(45.5 \%)$ & & \\
\hline & 1-4 days/week & & \\
\hline & $46(56.8 \%)$ & & \\
\hline & 35 (43.2\%) & & \\
\hline
\end{tabular}




\begin{tabular}{|c|c|c|c|}
\hline & Frequency & \multirow{3}{*}{$\begin{array}{l}\text { Statistical } \\
\text { Value } \\
\text { Pearson's } \\
\text { chi- } \\
\text { squared }\end{array}$} & \multirow[t]{3}{*}{$\begin{array}{l}\text { Level of } \\
\text { Significance }\end{array}$} \\
\hline & $\begin{array}{l}\text { Watch television, videos or play video } \\
\text { games (Less than } 2 \text { hours/ } 2 \text { or more } \\
\text { hours) }\end{array}$ & & \\
\hline & $\mathbf{N}(\%)$ & & \\
\hline & None & & \\
\hline & $42(53.2 \%)$ & & \\
\hline & $37(46.8 \%)$ & & \\
\hline \multirow[t]{15}{*}{ Breakfast classification } & Sweets, pastries and salty snacks & 0.78 & $p=0.999$ \\
\hline & $8(57.1 \%)$ & & \\
\hline & $6(42.9 \%)$ & & \\
\hline & Red, processed and could meats & & \\
\hline & $17(54.8 \%)$ & & \\
\hline & $14(45.2 \%)$ & & \\
\hline & Milk, lean meats, legumes, nuts, eggs & & \\
\hline & $87(55.4 \%)$ & & \\
\hline & $70(44.6 \%)$ & & \\
\hline & Vegetables and fruits & & \\
\hline & $62(56.4 \%)$ & & \\
\hline & $48(43.6 \%)$ & & \\
\hline & Bread, pasta, rice, potatoes & & \\
\hline & $28(57.1 \%)$ & & \\
\hline & $21(42.9 \%)$ & & \\
\hline \multirow{6}{*}{$\begin{array}{l}\text { Watch television at } \\
\text { lunch or dinner }\end{array}$} & Everyday & 1.118 & $p=0.773$ \\
\hline & $147(56.5 \%)$ & & \\
\hline & $113(43.5 \%)$ & & \\
\hline & 4-6 days/week & & \\
\hline & $23(56.1 \%)$ & & \\
\hline & $18(43.9 \%)$ & & \\
\hline
\end{tabular}




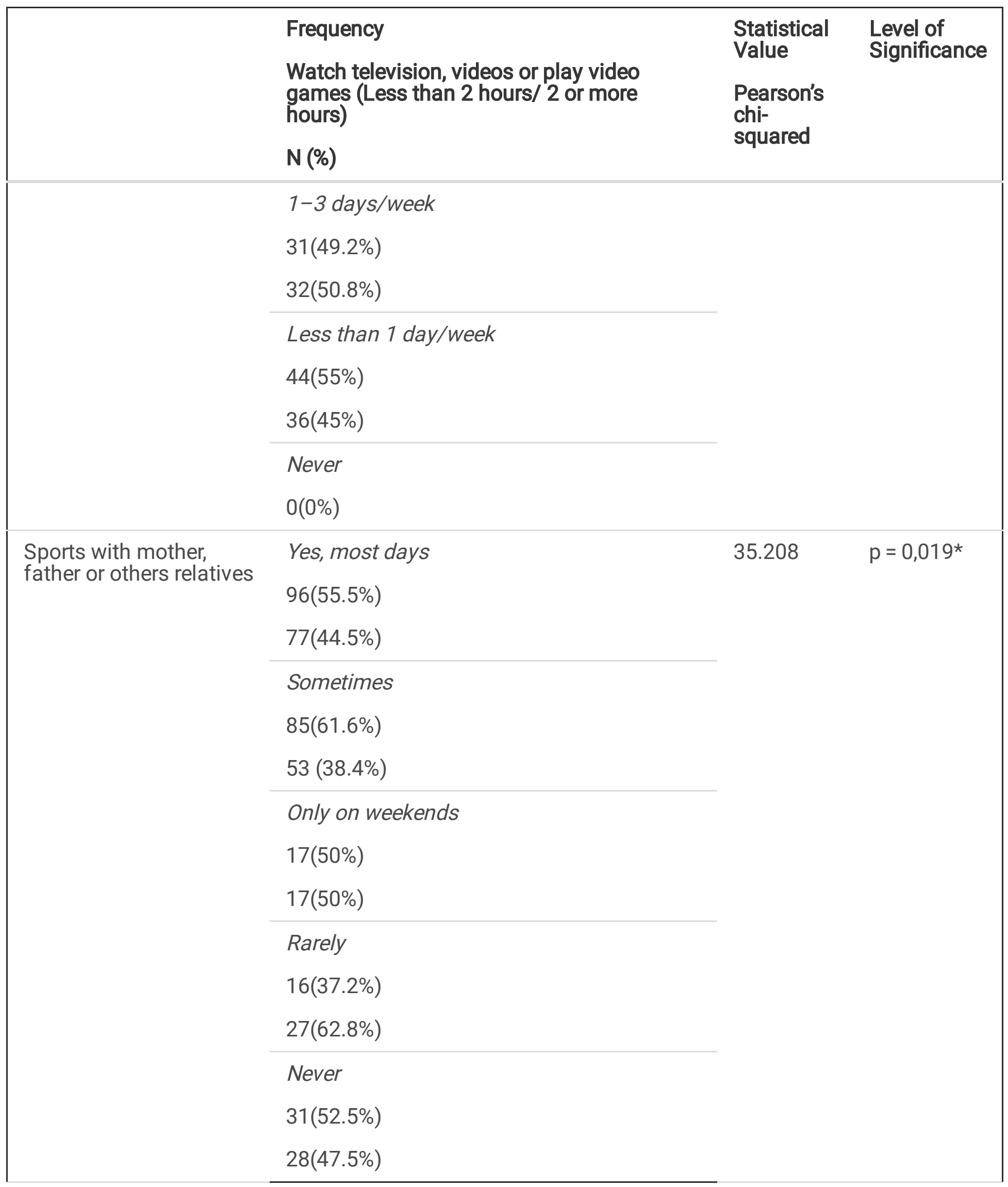


In the present study, a descriptive analysis of the data was carried out and various sociodemographic hypotheses were tested, as well as dietary habits and sedentary habits compared to the number of hours they watched television and / or played video games. The National Student Health Survey, carried out in Brazil, showed that $78 \%$ of adolescents were exposed to at least two hours a day of television [10]. In our study, the population was school aged between 9 and 10 years and the percentage of boys and girls who watched television or played video games at least 2 hours a day was $55 \%$.

Stahlmann et al observed that children from single-parent families reported longer hours of television consumption $(p<0.001)$ [18]. In our study, no significant difference was observed in this regard $(p=$ 0.476). However, the highest percentage of boys and girls who watched television over two hours was represented by those who lived with their father and a new partner (66.6\%), followed by those who lived alone with their mother (46.7\%).

There is scientific evidence linking the number of hours that children spend watching television with the omission of important meals during the day such as breakfast ${ }^{11}$. Specifically, in our study, no significant differences were obtained between the number of hours watching television and the weekly frequency of breakfast. However, it was found that almost half of children, who do not have breakfast, use to watch television or play video games during over two hours a day.

In this study, only $18 \%$ of boys and girls had breakfast every day of the week, a low rate compared to other studies carried out in Argentina [19], Spain [20] and Canada [21], where 75\%, 77.5\% and 85.5\%, respectively, had breakfast daily. However, in the study carried out in Spain [20], 5.2\% had fresh juices or whole fruits for breakfast; on the contrary in our study, this breakfast was taken by $24.4 \%$. In the study carried out in Argentina ${ }^{19}, 16 \%$ had a low-quality breakfast, based on industrial pastries and sugary foodstuffs, while in Italy [22], 31.3\% of children had this type of breakfast. By comparison, among Uruguaiana schoolchildren, only $3.1 \%$ had industrial pastries or sugary foodstuffs for breakfast. This was particularly noteworthy so we can conclude that there were fewer children who used to have the habit of a daily breakfast but those who did, ate a healthier breakfast, mainly based on milk, bread, eggs and fruit or fresh fruit juices. The problem in this case was the lack of the breakfast habit, which may be related to educational and socioeconomic factors due to scarcity of resources.

In previous studies, watching television was associated with a higher intake of "fast food", foodstuffs and foods rich in saturated fat, refined sugar, food colours and preservatives [23, 24, 25]. Overexposure to television and video games, in addition to leading a sedentary lifestyle, exposes children to the advertising of unhealthy products [26, 27]. In this study, no statistical significance was obtained between the number of hours spending watching television or playing video games and the type of breakfast that children used to have.

Statistical significance was obtained between the time spent watching television or playing video games and the frequency practising sports activities or physical exercise with their parents or other caregivers. This finding is similar to other ones found in previous studies, in which the important role of the family 
appears to be related to sedentary behaviours of children [28, 29, 30, 31]. In fact, parents' lifestyle habits are the main predictors of the time spent watching television by schoolchildren [32, 33, 34]. Bjelland et al. concluded that parental support and also their teaching and accompaniment in physical exercise decreased the time spent watching television or playing video games among European children $[35,36$, $37,38]$.

Another of the determining factors related to television and video game consumption is age and gender $[39,40]$. Regarding age, no differences were observed in this study, because there was hardly any variability, but on the other hand, there was a significant association between the times spent watching television and gender. Boys were more likely to watch over two hours on television or playing video games during the day than girls, as around $60 \%$ of them watched television during less than two hours a day.

\section{Limitations}

The intention of this study was to evaluate the sedentary behaviours of schoolchildren and their correlation with various sociodemographic and lifestyle factors. Children were asked to fill in the data related to his/her weight and height fin order to determinate their body mass index. There was a very low response rate because children uncovered these data. The body mass index could have been compared to sedentary habits, if we had had a higher response rate. Therefore, as a proposal for improvement for the next study, it is recommended personally size and weigh the children. It is also intended to include parents to expand the variables, as well as to contrast the information reported by the scholars.

\section{Conclusion}

In this study, an important finding was obtained, the association between the times spent watching television or playing video game and the practice of physical exercise in the family. Once again the importance of the family in education for the health of children is highlighted. The school environment provides direct access to schoolchildren and their parents to launch numerous health education programs based on physical exercise and a healthy diet.

\section{Declarations}

\section{Ethics approval and consent to participate}

The research obtained documentary authorization from the Regional Coordinating Office for Education of Rio Grande do Sul and from the Research Ethics Committee of the University of Unipampa. During the whole process of data collection, children's anonymity was guaranteed and the ethical principles for medical research in human beings described in the latest revision of the Declaration of Helsinki were applied.

\section{Consent for publication}


Not applicable.

\section{Availability of data and materials}

The datasets used and/or analyzed during the current study are available from the corresponding author on reasonable request.

\section{Competing interests}

The authors declare that they have no competing interests.

\section{Funding}

Not applicable.

\section{Authors' contributions}

All persons listed as authors have a thorough domain of the final manuscript, are able to defend its conclusions and have performed the following tasks. JAPB, JML and DRV participated in the conception and design of the study and drafted the first version of it. RRC, MEDV and JAPB participated in the development of the study and analysis of data. JML and JMRR revised critically the draft of the manuscript, with a key intellectual contribution to the final version. MEDV and DRV participated in the data collection and next to JAPB and RRC they made the final version of the manuscript.

\section{References}

1. WHO. Global Strategy on Diet, Phsysical Activity and Health. 2016. https://www.who.int/dietphysicalactivity/childhood/en/.

2. NCD Risk Factor Collaboration. Trends in adult body-mass index in 200 countries from 1975 to 2014: a pooled analysis of 1698 population-based measurement studies with 19.2 million participants. Lancet. 2016;387:1377-96.

3. Sahoo K, Sahoo B, Kumar A, Yasin N, Kumar R, Singh A. Childhood obesity: causes and consequences. J Family Med Prim Care. 2015;4:187-92.

4. Emond JA, Longacre MR, Drake KM, Titus LJ, Hendricks K, MacKenzie T, et al. Influence of childtargeted fast food TV advertising exposure on fast food intake: A longitudinal study of preschool-age children. Appetite. 2019;140:134-41.

5. Onis M. Prevenção do sobrepeso e da obesidade infantis. J Pediatr. 2015;91:105-7.

6. Dias da Rosa-dos. Santos F, Bueno-Vitola C, de Oliveira-Arriera C, da Silveira-Chagas MC, CalcagnoGomes G, Weiss-Pereira F. Ações de enfermeiros e professores na prevenção e no combate à obesidade infantil. Rev Rene. 2014; 15:463-70.

7. Borfe L, Rech DC, Schulz-Benelli TE, Nunes-Paiva D, Hedwing-Pohl H, Burgos MS. Associação entre a obesidade infantil e a capacidade cardiorrespiratória: revisão sistemática. Revista Brasileira em 
Promoção da Saúde. 2017; 30:118-24.

8. Dias-Amaral CM, de Oliveira JRG, Aparecido-Sampaio A. Estratégias e ações na prevenção da obesidade em escolares. Lecturas: Educación Física y Deportes. 2016; 21. [Revista digital] https://www.efdeportes.com/efd222/estrategias-e-acoes-na-prevencao-da-obesidade.htm.

9. Pedroso-Dias PJ, Prado-Domingos I, Gonçalves-Ferreira M, Muraro AP, Sichieri R, Veras-GonçalvesSilva RM. Prevalence and factors associated with sedentary behavior in adolescents. Rev Saúde Pública. 2014;48:266-74.

10. Guerra PH, Farias-Junior JC, Florindo AA. Comportamento sedentário em crianças e adolescentes brasileiros: revisão sistemática. Rev Saúde Pública. 2016; 50. [online] https://doi.org/10.1590/S1518-8787.2016050006307.

11. De Pinho L, Fagundes-Silveira M, de Carvalho-Botelho AC, Prates-Caldeira A. Identification of dietary patterns of adolescents attending public schools. J Pediatr. 2014;90:267-72.

12. Amigo-Vázquez I, Busto-Zapico R, Errasti-Pérez JM, Peña-Suárez E. Skipping breakfast, sedentarism and overweight in childre. Psychol Health Med. 2016;21:819-26.

13. Ministério Da Saúde. Política Nacional de Alimentação e Nutrição. 2013.

14. Sliwa SA, Sharma S, Dietz WH, Dolan PR, Nelson ME, Newman MB, Rockeymoore M, Economos CD. Healthy kids out of schools: using mixed methods to develop principles for promoting healthy eating and physical activity in out-of-school settings in the United States. Prev Chronic Dis. 2014;11:140207. DOI:http://dx.doi.org/10.5888/pcd11.140207.

15. Zamora-Corrales I, Jensen ML, Vandevijvere S, Ramírez-Zea M, Kroker-Lobos MF. Television food and beverage marketing to children in Costa Rica: current state and policy implications. Public Health Nutr. 2019;22:2509-20.

16. Vereecken C, Dupuy M, Rasmussen M. Breakfast consumption and its socio-demographic and lifestyle correlates in schoolchildren in 41 countries participating in the HBSC study. Int J Public Health. 2009;54:180-90.

17. World Medical Association

WMA Declaration of Helsinki-Ethical principles for medical research involving human subjects World Medical Association. WMA Declaration of Helsinki-Ethical principles for medical research involving human subjects. https://www.wma.net/policies-post/wma-declaration-of-helsinki-ethicalprinciples-for-medical-research-involving-human-subjects/. 2013.

18. Stahlmann K, Hebestreit A, DeHenauw S, Hunsberger M, Kaprio J, Lissner L, et al. A cross-sectional study of obesogenic behaviours and family rules according to family structure in European children. Int J Behav Nutr Phys Act. 2020;17:32. Doi.org/10.1186/s12966-020-00939-2.

19. Fugas V, Berta E, Walz F, Fortino MA, Martinelli MI. Hábito y calidad del desayuno en alumnos de dos escuelas primarias públicas de la ciudad de Santa Fe. Arch Argent Pediatr. 2013;111:502-7.

20. González-Rodríguez A, García-Padilla FM, Martos-Cerezuela I, Silvano-Arranz A, Fernández-Lao I. Proyecto ANDALIES: consumo, oferta y promoción de la alimentación saludable en los centros de educación secundaria de Andalucía. Nutr Hosp. 2015;31:1853-62. 
21. Lillico HG, Hammond D, Manske S, Murnaghan D. The prevalence of eating behaviors among Canadian youth using cross-sectional school-based surveys. BMC Public Health. 2014;14:1-12.

22. Nardone P, Lauria L, Buoncristiano M, Pizzi E, Galeone D, Spinelli A. I comporamenti alimentari dei bambini della scuola primaria in Italia fotografati dal Sistema di sorveglianza nazionale okkio alla salute. Epidemiol Prev. 2015;39:380-5.

23. Lee B, Kim H, Lee SK, Yoon J, Chung SJ. Effects of exposure to television advertising for energydense/ nutrient-poor food on children's food intake and obesity in South Korea. Appetite. 2014;81:305-11.

24. Emond JA, Longacre MR, Drake KM, Titus LJ, Hendricks K, MacKenzie T, et al. Influence of childtargeted fast food TV advertising exposure on fast food intake: A longitudinal study of preschool-age childre. Appetite. 2019;140:134-41.

25. Potvin Kent M, Pauzé $E$. The effectiveness of self-regulation in limiting the advertising of unhealthy foods and beverages on children's preferred websites in Canada. Public Health Nutr. 2018;21(9):1608-17.

26. Ponce-Blandón JA, Pabón-Carrasco M, Lomas-Campos MM. Análisis de contenido de la publicidad de productos alimenticios dirigidos a la población infantil. Gac Sanit. 2017;31:180-86.

27. Dixon H, Niven P, Scully M, Wakefield M. Food marketing with movie caracter toys: Effects on young children's preferences for unhealthy and healthier fast food meals. Appetite. 2017;117:342-50.

28. Salmon J, Timperio A, Telford A, Carver A, Crawford D. Association of family environment with children's television viewing and with low level of physical activitiy. Obes Res. 2005;13:1939-51.

29. Hennessy E, Hughes SO, Goldberg JP, Hyatt RR, Economos CD. Parent-child interactions and objectively measured child physical activity: a cross-sectional study. Int J Behav Nutr Phys Act. 2010;7:71.

30. Neshteruk CD, Nezami BT, Nino-Tapias G, Davison KK, Ward DS. The influence of fathers on children's physical activity: a review of the literature from 2009 to 2015. Prev Med. 2017;102:12-9.

31. Petersen TL, Moller LB, Brond JC, Jepsen R, Grontved A. Association between parent and child physical activity: a systematic review. Int J Behav Nutr Phys Act. 2020;17:67.

32. Rollo S, Gaston A, Prapavessis H. Cognitive and motivational factors associated with sedentary behavior: a systematic review. AIMS Public Health. 2016;3:956-84.

33. Pearce MS, Basterfield L, Mann KD, Parkinson KN, Adamson AJ, Reilly JJ: Early predictors of objectively measured physical activity and sedentary behaviour in 8-10 year old children: the Gateshead Millennium Study. PLoS One. 2012, 7: e37975-10.1371/journal.pone.0037975.

34. Xu C, Quan M, Zhang H, Zhou C, Chen P. Impact of parents' physical activity on preschool children's physical activity: a cross-sectional study. PeerJ. 2018;2:e4405.

35. Bjelland M, Soenens B, Bere E, Kovács É, Lien N, Maes L, Manios Y, Moschonis G. VeldeSJ: associations between parental rules, style of communication and children's screen time. BMC Public Health. 2015;15:1002. Doi.org/10.1186/s12889-015-2337-61. 
36. Pearson N, Salmon J, Crawford D, Campbell K, Timperio A. Are parental concerns for child TV viewing associated with child TV viewing and the home sedentary environment? Int J Behav Nutr Phys Act. 2011;8:102.

37. Abbott G, Salmon J, Timperio A, Kneebone K, Hnatiuk J, Hesketh K. Cross-sectional and longitudinal associations between parents' and preschoolers' physical activity and TV viewing. Obes Res Clin Pract. 2013;2:e113.

38. Butte NF, Gregorich SE, Tschann JM, Penilla C, Pasch LA, De Groat CL, Flores E, Deardorff J, Greenspan LC, Martínez SM. Longitudinal effects of parental, child and neighborhood factors on moderate-vigorous physical activity and sedentary time in Latino childre. Int J Behav Nutr Phys Act. 2014;11:108.

39. Janssen I, AG LB. Review Systematic review of the health benefits of physical activitiy and fitness in school-aged children and youth. Int J Behav Nutr Phys Act. 2010;7:1-16.

40. Foley L, Jiang Y, Mhurchu CN, Jull A, Prapavessis H, Rodgers A, Maddison R. The effect of active video games by ethnicity, sex and fitness: subgroup analysis from a randomised controlled trial. Int $\mathrm{J}$ Behav Nutr Phys Act. 2014;11:46.

\section{Figures}

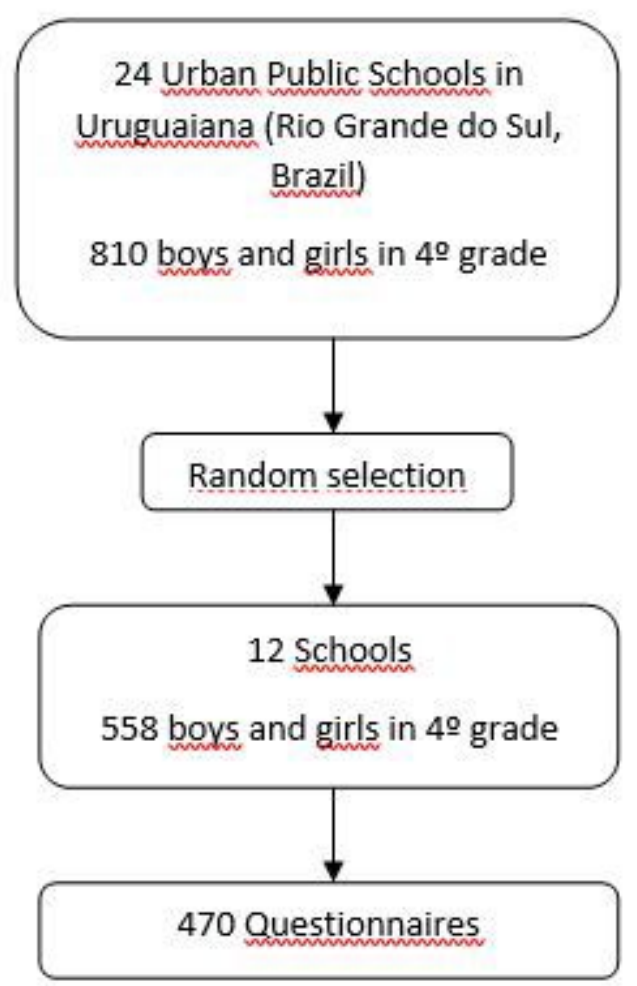

\section{Figure 1}

Sampling 


\section{Supplementary Files}

This is a list of supplementary files associated with this preprint. Click to download.

- STROBEchecklistcrosssectional1.doc 